\title{
Ordinal Efficiency and Dominated Sets of Assignments*
}

\author{
Atila Abdulkadiroğlu \\ Department of Economics, Columbia University, New York, New York 10027 \\ aa2061@columbia.edu \\ and \\ Tayfun Sönmez \\ Department of Economics, Koç University, 80910 Sartyer, Istanbul, Turkey \\ tsonmez@ku.edu.tr
}

\begin{abstract}
Using lotteries is a common tool for allocating indivisible goods. Since obtaining preferences over lotteries is often difficult, real-life mechanisms usually rely on ordinal preferences over deterministic outcomes. Bogomolnaia and Moulin [3] show that the outcome of an ex-post efficient mechanism may be stochastically dominated. They define a random assignment to be ordinally efficient if and only if it is not stochastically dominated. In this paper we investigate the relation between ex-post efficiency and ordinal efficiency. We introduce a new notion of domination defined over sets of assignments and show that a lottery induces an ordinally efficient random assignment if and only if each subset of the full support of the lottery is undominated. Journal of Economic Literature Classification Numbers: C71, C78, D71, D78.
\end{abstract}

Keywords: Random assignment, matching, ordinal efficiency, house allocation.

*We thank Ronald Jones, Biung-Ghi Ju, Tarık Kara, Hyungjun Kim, Semih Koray, Lionel McKenzie, Murat Sertel, William Thomson, İnsan Tunal, Lin Zhou, an anonymous referee, seminar participants at Bilkent, Boğaziçi, Caltech, Carnegie-Mellon, City University of Hong Kong, Maryland, Northwestern, Rochester, UCSB, USC and especially to Asher Wolinsky for their constructive comments. Abdulkadiroğlu gratefully acknowledges the Sloan Dissertation Fellowship and Sönmez gratefully acknowledges the research support of National Science Foundation via grant SES-9904214. All errors are our own responsibility. 
Proposed Running Head: Ordinal Efficieny

Correspondent:

Tayfun Sönmez, Department of Economics, Koç University, Rumelifeneri Yolu, Sarıer, 80910, İstanbul, Turkey

Phone: 90-532-5838861

Fax: 90-212-3381653

E-mail: tsonmez@ku.edu.tr 


\section{Introduction}

A house allocation problem ${ }^{1}$ is a resource allocation problem where a set of indivisible objects, say houses, has to be assigned to a set of agents. Some real-life applications include oncampus housing, public housing, office space assignment and parking space assignment.

Lotteries are widely used to restore fairness in house allocation problems. In particular random priority (also known as the random serial dictatorship), is very popular in real-life applications: Randomly order the agents and assign the first agent her top choice, the next agent her top choice among remaining houses, and so on. An important feature of this mechanism is that it merely relies on ordinal preferences over deterministic outcomes (i.e., houses) but not the preferences over lotteries. Indeed, in most applications it is unrealistic to expect agents to reveal their preferences over lotteries. So this feature of random priority is a major reason for its popularity in real-life applications.

Using a lottery restores fairness but how should one evaluate efficiency of these lotteries? Until recently the literature focused on ex-post efficiency when only ordinal preferences are available. Bogomolnaia and Moulin [3] recently provide an example in which the random priority, despite its ex-post efficiency, results in unambigious efficiency loss. ${ }^{2}$ In their example the random assignment that is induced by this mechanism is stochastically dominated by another feasible random assignment. Based on this example, they defined a random assignment to be ordinally efficient if and only if it is not stochastically dominated by another random assignment.

Ordinal efficiency is a stronger requirement than ex-post efficiency. Bogomolnaia and Moulin [3] study these notions from a mechanism design perspective. We, on the other hand, do not consider mechanism design aspect of the problem. Our main purpose is to understand the relation between these two notions of efficiency. In particular, we would like to understand why ex-post efficient lotteries might induce ordinally inefficient random assignments.

We start our quest to understand how these efficiency notions are related with a surprising observation: An ex-post efficient lottery may induce the same random assignment with an ex-post inefficient lottery (Example 2). In that sense an ex-post efficient lottery may be equivalent to an ex-post inefficient lottery and this equivalence provides at least a partial explanation to why ex-post efficient lotteries might induce ordinally inefficient ran-

\footnotetext{
${ }^{1}$ Hylland and Zeckhauser [12] initiate the literature on house allocation problems. See Zhou [24], Abdulkadiroğlu and Sönmez [1, 2], Svensson [23], Ehlers [6], Ergin [7], Papai [17], Bogomolnaia and Moulin [3], Miyagawa [16] for some recent results and Shapley and Scarf [22] for the closely related class of housing markets.

${ }^{2}$ Bogomolnaia and Moulin [4] show that a similar phemomenon happens in a related model where (1) objects are ranked the same way by all agents, (2) receiving no object is possible and (3) agents might consider different objects desirable. This model was initially introduced and studied by Crès and Moulin [5].
} 
dom assignments. Nevertheless this potential equivalence is not the main reason (Example 3 ). We introduce a domination notion which compares sets of assignments and show that randomizing between the members of a dominated set is responsible from this phenomenon (Theorem 1). Based on this result, we feel that our domination notion is the primary contribution of this paper. It is an extension of Pareto domination: Any set which consists of Pareto inefficient assignments is dominated. But more importantly, sets which consist of only Pareto efficient assignments might still be dominated.

An implication of our paper is the following: Use of social choice correspondances in social choice theory as well as in mechanism design is not uncommon. One interpretation of the use of social choice correspondances is that the eventual outcome will be chosen with a lottery. Requiring these correspondences not to contain dominated sets of outcomes would be a sensible normative criterion.

\section{The Model}

A house allocation problem is a triple $(N, H, \succ)$ where $N=\{1,2, \ldots, n\}$ is a finite set of agents, $H=\left\{h_{1}, h_{2}, \ldots, h_{n}\right\}$ is a finite set of objects (say houses) of the same cardinality with the set of agents and $\succ=\left(\succ_{1}, \succ_{2}, \ldots, \succ_{n}\right)$ is a list of preferences. Here $\succ_{i}$ is the strict preference relation of agent $i$ over $H$. Let $\succeq_{i}$ denote the weak preference relation and $\sim_{i}$ denote the indifference relation induced by $\succ_{i}$. Note that we have $h \sim_{i} h^{\prime}$ if and only if $h=h^{\prime}$.

An assignment is a function $\mu: N \rightarrow H$. Given a house $h$, let $\mu^{-1}(h) \equiv\{i \in N: \mu(i)=$ $h$ \} denote the set of agents who are assigned house $h$ under $\mu$. Let $\mathcal{M}$ denote the set of all assignments. A feasible assignment is an assignment $\mu$ such that

$$
\forall i, i^{\prime} \in N \quad i \neq i^{\prime} \Rightarrow \mu(i) \neq \mu\left(i^{\prime}\right) \text {. }
$$

Equivalently a feasible assignment is a bijection from $N$ to $H$. Let $\mathcal{M}^{f}$ denote the set of all feasible assignments.

A feasible assignment can be also represented as a permutation matrix $\pi$ (a $n \times n$ matrix with entries 0 or 1 and exactly one non-zero entry per row and one per column) where each row represents an agent and each column represents a house. Let $\Pi$ denote the set of all permutation matrices.

An assignment $\mu \in \mathcal{M}$ Pareto dominates another assignment $\nu \in \mathcal{M}$ if (1) $\mu(i) \succeq_{i} \nu(i)$ for all $i \in N$ and $(2) \mu(i) \succ_{i} \nu(i)$ for some $i \in N$. A feasible assignment is Pareto efficient if it is not Pareto dominated by any other feasible assignment.

A random consumption is a probability distribution over $H$. Let $\triangle H$ denote the set of all random consumptions. A lottery is a probability distribution over feasible assignments. A lottery is denoted by $\mathcal{L}=\sum \alpha_{\mu} \circ \mu$ where $\alpha_{\mu} \in[0,1]$ is the probability weight of the 
feasible assignment $\mu \in \mathcal{M}^{f}$. Given $i \in N$, let $\mathcal{L}(i)$ denote the random consumption lottery $\mathcal{L}$ allocates to agent $i$. Let $\triangle \mathcal{M}^{f}$ denote the set of all lotteries. A lottery is ex-post efficient if it assigns positive weight to only Pareto efficient assignments.

Each lottery induces a random assignment $P=\left[p_{i h}\right]_{i \in N, h \in H}$ where $p_{i h} \in[0,1]$ is the probability that agent $i$ receives house $h$. Let $P_{i}$ denote the resulting random consumption for agent $i$. Note that $P_{i} \in \triangle H$.

Let $\pi(\mu)$ be the permutation matrix that represents the feasible assignment $\mu \in \mathcal{M}^{f}$. Then the random assignment that is induced by lottery $\sum \alpha_{\mu} \circ \mu$ is $P=\sum \alpha_{\mu} . \pi(\mu)$. Clearly,

$$
\forall i \in N, h \in H, \quad p_{i h} \geq 0, \quad \sum_{i^{\prime} \in N} p_{i^{\prime} h}=\sum_{h^{\prime} \in H} p_{i h^{\prime}}=1
$$

Given any random assignment $P$ which satisfies (1), by the well-known Birkhoff-von Neumann theorem there exists at least one lottery $\mathcal{L}$ that induces $P{ }^{3}$ In this case we say $\mathcal{L}$ is a decomposition of $P$. Two distinct lotteries $\mathcal{L}$ and $\mathcal{L}^{\prime}$ may induce the same random assignment $P{ }^{4}$ We refer to any pair of such lotteries as equivalent lotteries. Note that for any agent $i \in N$, the equivalent lotteries $\mathcal{L}$ and $\mathcal{L}^{\prime}$ allocate the same random consumption $\mathcal{L}(i)=\mathcal{L}^{\prime}(i)=P_{i}$ to agent $i$.

\subsection{Ordinal Efficiency}

Given a strict preference relation $\succ_{i}$ and a house $h$, let $U\left(\succ_{i}, h\right) \equiv\left\{h^{\prime} \in H: h^{\prime} \succeq_{i} h\right\}$ be the upper contour set of $h$ at $\succ_{i}$. Given a pair of random consumptions $P_{i}, Q_{i}$ and a strict preference relation $\succ_{i}, P_{i}$ stochastically dominates $Q_{i}$ under $\succ_{i}$ if and only if

$$
\forall h \in H, \quad \sum_{h^{\prime} \in U\left(\succ_{i}, h\right)} p_{i h^{\prime}} \geq \sum_{h^{\prime} \in U\left(\succ_{i}, h\right)} q_{i h^{\prime}} .
$$

That is, $P_{i}$ stochastically dominates $Q_{i}$ if and only if for any house $h$, the probability of receiving a house that is at least as good as $h$ is at least as large under $P_{i}$ than under $Q_{i}{ }^{5}$ Given a pair of distinct random assignments $P, Q$ and a preference list $\succ, P$ stochastically dominates $Q$ under $\succ$ if and only if $P_{i}$ stochastically dominates $Q_{i}$ under $\succ_{i}$ for all $i \in N$. A random assignment $P$ is ordinally efficient (Bogomolnaia and Moulin [3]) at $\succ$, if and only if it is not stochastically dominated by any other random assignment under $\succ$.

An important implication of the lack of ordinal efficiency (i.e., ordinal inefficiency) of a random assignment $P$ is the following: Suppose agents are expected utility maximizers.

\footnotetext{
${ }^{3}$ See also Remark 1 in Garratt and Qin [9].

${ }^{4}$ See Roth, Rothblum and Vande Vate [20] for a similar observation in the context of two-sided matching markets (Gale and Shapley [8]).

${ }^{5}$ For stochastic dominance, see also Karlin [13], Quirk and Saposnik [18], Hadar and Russell [10], MasColell, Whinston and Green [14] and Roth and Rothblum [19].
} 
Since $P$ is stochastically dominated by another random assignment $Q$, every agent $i$ weakly prefers $Q_{i}$ to $P_{i}$ and at least one of them strictly prefers $Q_{i}$ to $P_{i}$ no matter what their von Neumann-Morgenstern utility functions are. Therefore a lottery which induces an ordinally inefficient random assignment cannot be Pareto efficient whenever agents are expected utility maximizers. ${ }^{6}$ This also shows that every ex-ante efficient lottery induces an ordinally efficient random assignment. ${ }^{7}$

Even when agents are expected utility maximizers, expecting them to reveal their von Neumann-Morgenstern utility functions is often unrealistic. A common compromise is requiring ex-post efficiency rather than ex-ante efficiency. Therefore an important question is whether an ex-post efficient lottery necessarilly induces an ordinally efficient random assignment. Bogomolnaia and Moulin [3] show that the answer is negative. More specifically they show that although the popular real life mechanism random priority is ex-post efficient, the random assignment it induces might be ordinally inefficient. ${ }^{8}$

Example 1 (Bogomolnaia and Moulin [3]): Let $N=\{1,2,3,4\}$ be the set of agents and $H=\{a, b, c, d\}$ be the set of houses. Preferences are as follows:

$$
\begin{array}{lll}
\text { agent 1: } a \succ_{1} b \succ_{1} c \succ_{1} d & \text { agent 3: } b \succ_{3} a \succ_{3} d \succ_{3} c \\
\text { agent 2: } a \succ_{2} b \succ_{2} c \succ_{2} d & \text { agent 4: } b \succ_{4} a \succ_{4} d \succ_{4} c
\end{array}
$$

Random priority randomly orders the agents with uniform distribution and the first agent receives her top choice, the next agent receives her top choice among the remaining houses, and so on. This mechanism always selects an ex-post efficient lottery and it induces the following random assignment for this problem:

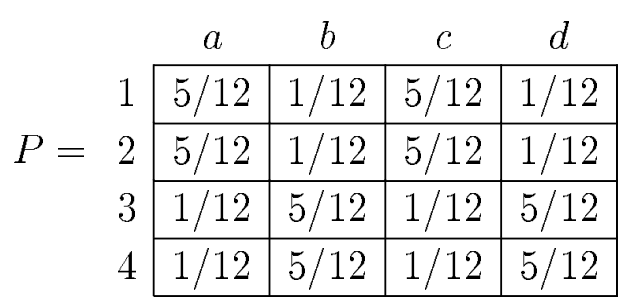

Next consider the following lottery

$$
\mathcal{L}=0.5\left(\begin{array}{llll}
1 & 2 & 3 & 4 \\
a & c & b & d
\end{array}\right)+0.5\left(\begin{array}{llll}
1 & 2 & 3 & 4 \\
c & a & d & b
\end{array}\right)
$$

which induces the random assignment

\footnotetext{
${ }^{6}$ This is not to say that ordinal efficiency is appealing only when agents are expected utility maximizers. Indeed it is difficult to imagine any natural preference relation over $\triangle H$ where an agent prefers a random consumption to another one which stochastically dominates it.

${ }^{7}$ McLennan [15] proves a converse statement which was conjectured in Bogomolnaia and Moulin [3]: Each ordinally efficient random assignment is induced by an ex-ante efficient lottery.

${ }^{8}$ Bogomolnaia and Moulin [3] also show that an ex-post efficient lottery necessarilly induces an ordinally efficient random assignment whenever there are at most 3 agents.
} 


\begin{tabular}{|c|c|c|c|c|}
\hline & $a$ & $b$ & $c$ & $d$ \\
\hline 1 & $1 / 2$ & 0 & $1 / 2$ & 0 \\
\hline$Q=2$ & $1 / 2$ & 0 & $1 / 2$ & 0 \\
\hline 3 & 0 & $1 / 2$ & 0 & $1 / 2$ \\
\hline 4 & 0 & $1 / 2$ & 0 & $1 / 2$ \\
\hline
\end{tabular}

Note that the random assignment $P$ assigns everyone their top choices with $5 / 12$ probability, second choices with $1 / 12$ probability, third choices with 5/12 probability and last choices with $1 / 12$ probability whereas $Q$ assigns everyone their top choices with $1 / 2$ probability and third choices with $1 / 2$ probability. Hence $Q$ stochastically dominates $P$ and therefore $P$ is ordinally inefficient.

Bogomolnaia and Moulin [3] also characterize the class of all ordinally efficient random assignments by a natural constructive algorithm. Think of each house as 1 unit of an infinitely divisible commodity where "agent $i$ receives $p \in[0,1]$ units of house $h$ " means she receives house $h$ with probability $p$. Each agent is given an exogenous eating speed function, specifying a rate of instant consumption for each time $t \in[0,1]$ such that the integral of each function is 1 . Given a profile of preferences, their algorithm works as follows: Each agent "eats" from her best available house at the given speed, where a house is available at time $t$ if and only if less than 1 unit has been eaten away up to time $t$ by all agents.

\subsection{Equivalent Lotteries and Ex-post Efficiency}

Example 1 (Bogomolnaia and Moulin [3]) makes a very important point. Expecting agents to reveal their preferences over lotteries is often unrealistic (not only in the present context but in other resource allocation problems as well). As a result, agents are often asked to reveal their ordinal preferences over deterministic outcomes. Until very recently the literature considered ex-post efficiency the main efficiency notion when only ordinal preferences are available. Bogomolnaia and Moulin [3] shows that an ex-post efficient outcome might still result in unambigious efficiency loss. One of our purposes in this paper is to understand why ex-post efficient lotteries induce ordinally inefficient random assignments.

A natural question at this point is the following: Consider a pair of equivalent lotteries $\mathcal{L}, \mathcal{L}^{\prime}$ and suppose $\mathcal{L}$ is ex-post efficient. Does that imply that $\mathcal{L}^{\prime}$ is also ex-post efficient? If not, that means the random assignment $P$ which is induced by $\mathcal{L}$ is not ordinally efficient and hence provides at least a partial understanding of why ex-post efficient lotteries might induce ordinally inefficient random assignments.

Example 2 (Two equivalent lotteries, one ex-post efficient - the other not): Let $N=\{1,2,3,4\}$ be the set of agents and $H=\{a, b, c, d\}$ be the set of houses. Preferences 
are as follows:

$$
\begin{aligned}
& \text { agent 1: } a \succ_{1} b \succ_{1} c \succ_{1} d \quad \text { agent } 3: b \succ_{3} a \succ_{3} d \succ_{3} c \\
& \text { agent 2: } a \succ_{2} b \succ_{2} c \succ_{2} d \quad \text { agent } 4: \quad b \succ_{4} a \succ_{4} d \succ_{4} c
\end{aligned}
$$

Consider the following random assignment:

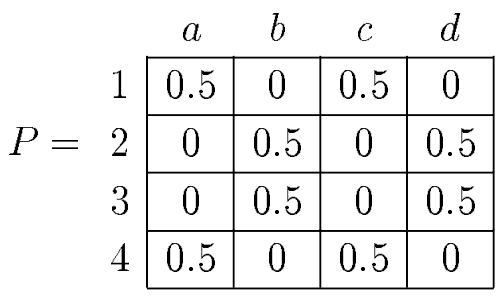

$P$ has two decompositions:

$$
\mathcal{L}=0.5\left(\begin{array}{llll}
1 & 2 & 3 & 4 \\
a & b & d & c
\end{array}\right)+0.5\left(\begin{array}{llll}
1 & 2 & 3 & 4 \\
c & d & b & a
\end{array}\right)
$$

and

$$
\mathcal{L}^{\prime}=0.5\left(\begin{array}{llll}
1 & 2 & 3 & 4 \\
a & d & b & c
\end{array}\right)+0.5\left(\begin{array}{llll}
1 & 2 & 3 & 4 \\
c & b & d & a
\end{array}\right)
$$

Among the two $\mathcal{L}$ is ex-post efficient but $\mathcal{L}^{\prime}$ is not. Indeed $\mathcal{L}^{\prime}$ is a lottery between exclusively Pareto inefficient assignments.

At this point we have at least a partial understanding of why ex-post efficient lotteries might induce ordinally inefficient random assignments. A natural next question is the following: Suppose not only a lottery is ex-post efficient, but also all its equivalent lotteries as well. Does that imply that the induced random assignment is ordinally efficient? If the answer is positive, then the potential equivalence between ex-post efficient and ex-post inefficient lotteries provides us a complete understanding of why ex-post efficient lotteries might induce ordinally inefficient random assignments. It turns out that the answer to this question is negative.

Example 3 (An ex-post efficient lottery with no equivalent lottery and which induces an ordinally inefficient random assignment): Let $N=\{1,2,3,4,5\}$ be the set of agents and $H=\{a, b, c, d, e\}$ be the set of houses. Preferences are as follows:

$$
\begin{aligned}
& \text { agent 1:c } \succ_{1} a \succ_{1} b \succ_{1} d \succ_{1} e \quad \text { agent } 4: a \succ_{4} d \succ_{4} b \succ_{4} c \succ_{4} e \\
& \text { agent 2:a } \succ_{2} b \succ_{2} e \succ_{2} c \succ_{2} d \quad \text { agent } 5: b \succ_{5} d \succ_{5} a \succ_{5} c \succ_{5} e \\
& \text { agent } 3: b \succ_{3} c \succ_{3} e \succ_{3} a \succ_{3} d
\end{aligned}
$$

Consider the following random assignment: 


\begin{tabular}{|c|c|c|c|c|c|}
\hline & $a$ & $b$ & $c$ & $d$ & $e$ \\
\hline 1 & 0.5 & 0 & 0.5 & 0 & 0 \\
\hline 2 & 0 & 0.5 & 0 & 0 & 0.5 \\
\hline 3 & 0 & 0 & 0.5 & 0 & 0.5 \\
\hline 4 & 0.5 & 0 & 0 & 0.5 & 0 \\
\hline 5 & 0 & 0.5 & 0 & 0.5 & 0 \\
\hline
\end{tabular}

$P$ has a unique decomposition: ${ }^{9}$

$$
\mathcal{L}=0.5\left(\begin{array}{lllll}
1 & 2 & 3 & 4 & 5 \\
a & e & c & d & b
\end{array}\right)+0.5\left(\begin{array}{lllll}
1 & 2 & 3 & 4 & 5 \\
c & b & e & a & d
\end{array}\right)
$$

$\mathcal{L}$ is an ex-post efficient lottery. Next consider the following lottery

$$
\mathcal{L}^{\prime}=0.5\left(\begin{array}{lllll}
1 & 2 & 3 & 4 & 5 \\
c & a & e & d & b
\end{array}\right)+0.5\left(\begin{array}{lllll}
1 & 2 & 3 & 4 & 5 \\
c & e & b & a & d
\end{array}\right)
$$

\begin{tabular}{|c|c|c|c|c|c|}
\hline \multirow{6}{*}{$Q=$} & $a$ & $b$ & $c$ & $d$ & $e$ \\
\hline & 0 & 0 & 1 & 0 & 0 \\
\hline & 0.5 & 0 & 0 & 0 & 0.5 \\
\hline & 0 & 0.5 & 0 & 0 & 0.5 \\
\hline & 0.5 & 0 & 0 & 0.5 & 0 \\
\hline & 0 & 0.5 & 0 & 0.5 & 0 \\
\hline
\end{tabular}

which induces

For $i=1,2,3$ the random consumption $Q_{i}$ stochastically dominates the random consumption $P_{i}$ and for $i=4,5$ the random consumption $Q_{i}$ is equal to the random consumption $P_{i}$. Therefore $P$ (which can only be induced by the expost-efficient lottery $\mathcal{L}$ ) is ordinally inefficient.

\subsection{Dominated Sets of Assignments}

We need some additional notation in order to introduce a new domination notion which will be of key importance for this paper.

A set of assignments $\widetilde{M} \subseteq \mathcal{M}$ is frequency equivalent to a set of assignments $M^{\prime} \subseteq \mathcal{M}$ with $\left|M^{\prime}\right|=|\widetilde{M}|$ if and only if

$$
\forall i \in N \quad \bigsqcup_{\mu \in \widetilde{M}} \mu(i)=\bigsqcup_{\mu \in M^{\prime}} \mu(i)
$$

\footnotetext{
${ }^{9}$ Let the permuation matrix $\pi$ be one of the bases for an arbitrary decomposition of $P$. Either $\pi_{11}=1$ or $\pi_{41}=1$. For the former case $\pi_{11}=1 \Rightarrow \pi_{13}=0 \Rightarrow \pi_{33}=1 \Rightarrow \pi_{35}=0 \Rightarrow \pi_{25}=1 \Rightarrow \pi_{22}=0 \Rightarrow \pi_{52}=1 \Rightarrow$ $\pi_{54}=0 \Rightarrow \pi_{44}=1$ imposing a unique permutation matrix. For the latter case $\pi_{41}=1 \Rightarrow \pi_{11}=0 \Rightarrow \pi_{13}=$ $1 \Rightarrow \pi_{33}=0 \Rightarrow \pi_{35}=1 \Rightarrow \pi_{25}=0 \Rightarrow \pi_{22}=1 \Rightarrow \pi_{52}=0 \Rightarrow \pi_{54}=1$ also imposing a unique permutation matrix. Therefore $\pi$ should be one of these two permutation matrices.
} 
where $\bigsqcup$ denotes the variant of the union operator which allows for replicated elements.

A set of feasible assignments $M^{\prime} \subseteq \mathcal{M}^{f}$ dominates a set of feasible assignments $M \subseteq \mathcal{M}^{f}$ if and only if

1. there exists a set of assignments $\widetilde{M} \subseteq \mathcal{M}$ that is frequency equivalent to $M^{\prime}$ and

2. there exists a one-to-one function $f: \widetilde{M} \rightarrow M$ such that

(a) for all $\mu \in \widetilde{M}, \mu$ Pareto dominates or equal to $f(\mu)$, and

(b) there exists $\mu \in \widetilde{M}$ such that $\mu$ Pareto dominates $f(\mu)$.

Remark 1: Suppose that a set of infeasible assignments $\widetilde{M}$ is frequency equivalent to a set of feasible assignments $M^{\prime}$. Since a lottery is defined to be a probability distribution over feasible assignments, the uniform probability distribution over $\widetilde{M}$ (i.e. $\sum_{\mu \in \widetilde{M}} \frac{1}{|\widetilde{M}|} \circ \mu$ ) is not a lottery. Nevertheless $\sum_{\mu \in \widetilde{M}} \frac{1}{|\widetilde{M}|} \circ \mu$ induces the same random assignment with the uniform lottery over $M^{\prime}$. This observation will be key to relate our domination notion with ordinal efficiency.

Clearly the singleton that consists of a feasible but Pareto inefficient assignment $\nu \in \mathcal{M}^{f}$ is dominated by the singleton that consists of a feasible assignment $\mu \in \mathcal{M}^{f}$ that Pareto dominates $\nu$. (Here $M^{\prime}=\widetilde{M}=\{\mu\}$ and $f(\mu)=\nu$.) Therefore our domination notion is an extension of Pareto domination.

What is more important is that a set of feasible and Pareto efficient assignments might still be dominated.

Example 3 (continued): Consider the random assignment problem in Example 3. Both assignments in the set

$$
M=\left\{\mu_{1}, \mu_{2}\right\}=\left\{\left(\begin{array}{lllll}
1 & 2 & 3 & 4 & 5 \\
a & e & c & d & b
\end{array}\right),\left(\begin{array}{lllll}
1 & 2 & 3 & 4 & 5 \\
c & b & e & a & d
\end{array}\right)\right\}
$$

are feasible and Pareto efficient. Next consider the sets

$$
\begin{gathered}
M^{\prime}=\left\{\mu_{1}^{\prime}, \mu_{2}^{\prime}\right\}=\left\{\left(\begin{array}{lllll}
1 & 2 & 3 & 4 & 5 \\
c & a & e & d & b
\end{array}\right),\left(\begin{array}{lllll}
1 & 2 & 3 & 4 & 5 \\
c & e & b & a & d
\end{array}\right)\right\} \text { and } \\
\widetilde{M}=\left\{\tilde{\mu}_{1}, \tilde{\mu}_{2}\right\}=\left\{\left(\begin{array}{lllll}
1 & 2 & 3 & 4 & 5 \\
c & e & b & d & b
\end{array}\right),\left(\begin{array}{lllll}
1 & 2 & 3 & 4 & 5 \\
c & a & e & a & d
\end{array}\right)\right\} .
\end{gathered}
$$

Note that (1) both elements of $M^{\prime}$ are feasible assignments, (2) $\widetilde{M}$ is frequency equivalent to $M^{\prime}$, and (3) $\tilde{\mu}_{1}$ Pareto dominates $\mu_{1}$ and $\tilde{\mu}_{2}$ Pareto dominates $\mu_{2}$. Therefore $M^{\prime}$ dominates $M$. 
Remark 2: Even though members of $M^{\prime}$ do not Pareto dominate members of $M$ on a one-by-one basis, members of $\widetilde{M}$ do. Therefore infeasible assignments play a key role in our dominance condition.

One could also consider the following stronger version of our domination notion: A set of feasible assignments $M^{\prime} \subseteq \mathcal{M}^{f}$ strictly dominates a set of feasible assignments $M \subseteq \mathcal{M}^{f}$ if and only if

1. there exists a set of assignments $\widetilde{M} \subseteq \mathcal{M}$ that is frequency equivalent to $M^{\prime}$ and

2. there exists a one-to-one function $f: \widetilde{M} \rightarrow M$ such that for all $\mu \in \widetilde{M}, \mu$ Pareto dominates $f(\mu)$.

Our first result states that these two notions are essentially equivalent.

Proposition 1: Let $M \subseteq \mathcal{M}^{f}$ be dominated. Then there exists $M^{*} \subseteq M$ that is strictly dominated.

Proof: See the Appendix.

\section{Main Characterization Result}

Ordinal efficiency and our domination notion are closely related. In order to explain this relation, we need additional notation.

Given a lottery $\mathcal{L}=\sum \alpha_{\mu} \circ \mu$, let $M(\mathcal{L}) \equiv\left\{\mu \in \mathcal{M}^{f}: \alpha_{\mu}>0\right\}$ be the full support of lottery $\mathcal{L}$.

What is wrong with a lottery which has a subset of its full support that is dominated? Loosely speaking, the flaw of such a lottery is similar to that of a lottery which gives positive weight to Pareto inefficient assignments. We illustrate this point with the following (familiar) example.

Example 3 (continued again): We have already shown that both assignments in $M$ are Pareto efficient and yet $M$ is dominated by $M^{\prime}$. Now consider the lottery $\mathcal{L}=0.5 \mu_{1}+0.5 \mu_{2}$. Had any of the assignments in $M$, say $\mu_{1}$, been Pareto inefficient, replacing it with a feasible assignment that Pareto dominates $\mu_{1}$ would result in a "better" lottery. Similarly had assignments $\tilde{\mu}_{1}, \tilde{\mu}_{2}$ been feasible, replacing $\mu_{1}$ with $\tilde{\mu}_{1}$ and $\mu_{2}$ with $\tilde{\mu}_{2}$ would result in a "better" lottery. That is because, $\tilde{\mu}_{1}$ Pareto dominates $\mu_{1}$ and $\tilde{\mu}_{2}$ Pareto dominates $\mu_{2}$. Unfortunately neither $\tilde{\mu}_{1}$ nor $\tilde{\mu}_{2}$ is feasible. Nevertheless, because $\widetilde{M}=\left\{\tilde{\mu}_{1}, \tilde{\mu}_{2}\right\}$ and $M^{\prime}=$ $\left\{\mu_{1}^{\prime}, \mu_{2}^{\prime}\right\}$ are frequency equivalent, agents do not care whether $\left[\mu_{1}\right.$ is replaced with $\tilde{\mu}_{1}$ and $\mu_{2}$ is replaced with $\left.\tilde{\mu}_{2}\right]$ or $\left[\mu_{1}\right.$ is replaced with $\mu_{1}^{\prime}$ and $\mu_{2}$ is replaced with $\left.\mu_{2}^{\prime}\right]$. Hence lottery $\mathcal{L}^{\prime}=0.5 \mu_{1}^{\prime}+0.5 \mu_{2}^{\prime}$ is a "better" lottery than $\mathcal{L}=0.5 \mu_{1}+0.5 \mu_{2}$.

We are now ready to generalize this observation. 
Proposition 2: Let $\mathcal{L}$ be an arbitrary decomposition of a random assignment $P$ and let $M$ be an arbitrary subset of the full support of lotter $\mathcal{L}$. If $M$ is dominated then $P$ is ordinally inefficient.

Proof: We construct a random assignment $Q$ which stochastically dominates $P$.

Let $M \subseteq M(\mathcal{L})$ be dominated by $M^{\prime} \subseteq \mathcal{M}^{f}$. Let $\epsilon=\min \left\{\alpha_{\mu}: \mu \in M\right\}$,

$$
\mathcal{L}^{\prime}=\mathcal{L}-\epsilon \cdot \sum_{\mu \in M} \mu+\epsilon \cdot \sum_{\mu \in M^{\prime}} \mu
$$

and $Q$ be the random assignment that is induced by lottery $\mathcal{L}^{\prime}$.

Recall that $M^{\prime}$ is frequency equivalent to a set of assignments $\widetilde{M}$ whose members Pareto dominate members of $M$ on a one-to-one basis. Therefore modifying lottery $\mathcal{L}$ by decreasing weights of members of $M$ by $\epsilon$ and replacing them by members of $M^{\prime}$ results in a lottery $\mathcal{L}^{\prime}$ such that $\mathcal{L}^{\prime}(i)$ stochastically dominates $\mathcal{L}(i)$ for each $i \in N$. Therefore the random assignment $Q$ that is induced by $\mathcal{L}^{\prime}$ stochastically dominates $P$.

It turns out that the converse statement holds as well. We need the following Lemma in order to prove this result.

Lemma 1: Let $\widetilde{M}=\left\{\tilde{\mu}_{1}, \tilde{\mu}_{2}, \ldots, \tilde{\mu}_{k}\right\} \subset \mathcal{M}$ be a set of assignments such that

$$
\forall h \in H \quad \sum_{t=1}^{k}\left|\tilde{\mu}_{t}^{-1}(h)\right|=k .
$$

Then there exists a set of feasible assignments $M^{\prime}=\left\{\mu_{1}^{\prime}, \mu_{2}^{\prime}, \ldots, \mu_{k}^{\prime}\right\} \subset \mathcal{M}^{f}$ such that $\widetilde{M}$ is frequency equivalent to $M^{\prime}$.

Proof: See the Appendix.

Theorem 1: Let $\mathcal{L}$ be an arbitrary decomposition of a random assignment $P$. $P$ is ordinally efficient if and only if each subset $M$ of the full support of lottery $\mathcal{L}$ is undominated.

Proof: Proof of the "only if" part follows from Proposition 2. We next prove the "if" part.

Let $P$ be an arbitrary random assignment. Suppose $P$ is ordinally inefficient. That means there exists a random assignment $Q$ which stochastically dominates $P$. Since both $P$ and $Q$ are random assignments, $Q-P$ is a zero-sum matrix (i.e. an $n \times n$ matrix where each row and each column adds up to zero). Then we can decompose $Q-P=A-B$ where the matrices $A=\left[a_{i h}\right]_{i \in N, h \in H}$ and $B=\left[b_{i h}\right]_{i \in N, h \in H}$ are such that

$$
a_{i h}=\left\{\begin{array}{cl}
q_{i h}-p_{i h} & \text { if } q_{i h}-p_{i h}>0 \\
0 & \text { otherwise }
\end{array} \quad \text { and } \quad b_{i h}=\left\{\begin{array}{cl}
p_{i h}-q_{i h} & \text { if } p_{i h}-q_{i h}>0 \\
0 & \text { otherwise }
\end{array}\right.\right.
$$


Then

$$
\begin{array}{ll}
\forall i \in N, h \in H & a_{i h}>0 \Rightarrow b_{i h}=0 \text { and } b_{i h}>0 \Rightarrow a_{i h}=0 \\
\forall i \in N, h \in H & a_{i h}>0 \Rightarrow \exists i^{\prime} \neq i \text { such that } b_{i^{\prime} h}>0 \\
\forall i \in N, h \in H & b_{i h}>0 \Rightarrow \exists h^{\prime} \in H \text { such that } a_{i h^{\prime}}>0 \text { and } h^{\prime} \succ_{i} h
\end{array}
$$

Here (2) follows by construction, (3) follows by construction together with the fact that $A-B=Q-P$ is a zero-sum matrix and (4) follows by definition.

Since $Q$ stochastically dominates $P$, there exists an agent-house pair $\left(\tilde{i}^{1}, \tilde{h}^{1}\right) \in N \times H$ such that $b_{\tilde{i}^{1} \tilde{h}^{1}}>0$. Moreover relation (4) implies there exists $\tilde{h}^{2} \in H$ such that $a_{\tilde{i}^{1} \tilde{h}^{2}}>0$ and $\tilde{h}^{2} \succ_{\tilde{i}^{1}} \tilde{h}^{1}$ and hence relation (3) implies there exists $\tilde{i}^{2} \in N$ such that $b_{\tilde{i}^{2} \tilde{h}^{2}}>0$.

Similarly, given a sequence of (not necessarilly distinct) agent-house pairs $\left(\tilde{i}^{1}, \tilde{h}^{1}\right),\left(\tilde{i}^{2}, \tilde{h}^{2}\right), \ldots,\left(\tilde{i}^{k}, \tilde{h}^{k}\right)$ with

1. $b_{\tilde{i}^{i}} \tilde{h}^{l}>0$ for $l=1,2, \ldots, k$ and

2. $\tilde{h}^{l+1} \succ_{\tilde{i}^{l}} \tilde{h}^{l}$ for $l=1,2, \ldots, k-1$

there exists another pair $\left(\tilde{i}^{k+1}, \tilde{h}^{k+1}\right) \in N \times H$ such that $b_{\tilde{i}^{k+1} \tilde{h}^{k+1}}>0$ and $\tilde{h}^{k+1} \succ_{\tilde{i}^{k}} \tilde{h}^{k}$. When we construct a sequence in this way, since there are $n^{2}$ distinct pairs, at some point a pair in this sequence will re-appear in the sequence. Therefore there exists a sequence ${ }^{10}$ $\left(i^{1}, h^{1}\right),\left(i^{2}, h^{2}\right), \ldots,\left(i^{k}, h^{k}\right)$ of distinct pairs such that

$$
\begin{aligned}
& b_{i^{l} h^{l}}>0 \text { for } l=1,2, \ldots, k \\
& h^{l+1} \succ_{i^{l}} h^{l} \text { for } l=1,2, \ldots, k-1 \text { and } h^{1} \succ_{i^{k}} h^{k}
\end{aligned}
$$

Relations (2) and (5) imply $a_{i^{l} h^{l}}=0$ for $l=1,2, \ldots, k$ and this together with $Q-A=P-B$ imply

$$
\forall l \in\{1,2, \ldots, k\} \quad p_{i^{l} h^{l}}-b_{i^{l} h^{l}}=q_{i^{l} h^{l}}-a_{i^{l} h^{l}}=q_{i^{l} h^{l}} \geq 0
$$

Therefore

$$
\forall l \in\{1,2, \ldots, k\} \quad p_{i^{l} h^{l}} \geq b_{i^{l} h^{l}}>0
$$

\footnotetext{
${ }^{10}$ Existence of such a sequence is also implied by Lemma 3 in Bogomolnaia \& Moulin [3] where they characterize the set of ordinally efficient random assignments by the acyclicity of the following binary relation on $\mathrm{H}$ :

$$
\forall h, h^{\prime} \in H \quad h \tau(P, \succ) h^{\prime} \Leftrightarrow\left\{\text { there exists } i \in N: h \succ_{i} h^{\prime} \text { and } p_{i h^{\prime}}>0\right\}
$$
}


Let $\mathcal{L}$ be an arbitrary decomposition of $P$. We want to show that there exists $M \subseteq M(\mathcal{L})$ that is dominated. Relation (7) implies that

$$
\forall l \in\{1,2, \ldots, k\} \quad \exists \mu \in M(\mathcal{L}) \text { such that } \mu\left(i^{l}\right)=h^{l}
$$

Next recursively construct a subset $M$ of $M(\mathcal{L})$ together with a function $g:\{1,2, \ldots, k\} \rightarrow$ $\{1,2, \ldots, k\}$ as follows:

1. For $l=1$, pick a feasible assignment $\mu_{1} \in M(\mathcal{L})$ such that $\mu_{1}\left(i^{1}\right)=h^{1}$. Set $M^{1}=\left\{\mu_{1}\right\}$ and $g(1)=1$.

2. Given $M^{l-1}$, if there is a feasible assignment $\mu_{t} \in M^{l-1}$ such that $\mu_{t}\left(i^{l}\right)=h^{l}$ then set $M^{l}=M^{l-1}$ and $g(l)=t$. Otherwise let $s=\left|M^{l-1}\right|+1$ and pick a feasible assignment $\mu_{s} \in M(\mathcal{L})$ such that $\mu_{s}\left(i^{l}\right)=h^{l}$; set $M^{l}=M^{l-1} \cup\left\{\mu_{s}\right\}$ and $g(l)=s$.

3. Set $M=M^{k}=\left\{\mu_{1}, \ldots, \mu_{k^{\prime}}\right\}$. Note that $k^{\prime} \leq k$.

By construction

$$
\forall l \in\{1,2, \ldots, k\} \quad \mu_{g(l)}\left(i^{l}\right)=h^{l}
$$

Let $g^{-1}:\left\{1, \ldots, k^{\prime}\right\} \rightarrow\{1, \ldots, k\}$ be the inverse correspondence of $g$. That is,

$$
\forall t \in\left\{1, \ldots, k^{\prime}\right\} \quad g^{-1}(t) \equiv\{l \in\{1, \ldots, k\}: g(l)=t\}
$$

Now construct the set of assignments $\widetilde{M}=\left\{\tilde{\mu}_{1}, \ldots, \tilde{\mu}_{k^{\prime}}\right\}$ as follows: For all $t \in$ $\left\{1, \ldots, k^{\prime}\right\}$,

$$
\begin{aligned}
& \forall l \in\{1, \ldots, n\} \backslash g^{-1}(t) \quad \tilde{\mu}_{t}\left(i^{l}\right)=\mu_{t}\left(i^{l}\right), \text { and } \\
& \forall l \in g^{-1}(t) \quad \begin{cases}\text { if } l<k & \text { then } \tilde{\mu}_{t}\left(i^{l}\right)=\mu_{g(l+1)}\left(i^{l+1}\right)=h^{l+1} \\
\text { if } l=k & \text { then } \tilde{\mu}_{t}\left(i^{l}\right)=\mu_{g(1)}\left(i^{1}\right)=h^{1}\end{cases}
\end{aligned}
$$

Note that

$$
\forall h \in H \quad \sum_{t=1}^{k^{\prime}}\left|\tilde{\mu}_{t}^{-1}(h)\right|=\sum_{t=1}^{k^{\prime}}\left|\mu_{t}^{-1}(h)\right|=k^{\prime}
$$

Therefore $\widetilde{M}$ is frequency equivalent to a set of feasible assignments $M^{\prime}=\left\{\mu_{1}^{\prime}, \ldots, \mu_{k^{\prime}}^{\prime}\right\}$ by Lemma 1. Moreover relations (6), (8), (9) and (10) imply that for all $t \in\left\{1, \ldots, k^{\prime}\right\}$,

$$
\begin{aligned}
& \forall l \in\{1, \ldots, n\} \backslash g^{-1}(t) \quad \tilde{\mu}_{t}\left(i^{l}\right) \sim_{i^{l}} \mu_{t}\left(i^{l}\right), \text { and } \\
& \forall l \in g^{-1}(t) \quad \underbrace{\tilde{\mu}_{t}\left(i^{l}\right)}_{h^{l+1}} \succ_{i^{l}} \underbrace{\mu_{t}\left(i^{l}\right)}_{h^{l}}
\end{aligned}
$$

Hence $M^{\prime}$ dominates $M \subset M(\mathcal{L})$ completing the proof. 


\section{A Appendix: Omitted Proofs}

Proposition 1: Let $M \subseteq \mathcal{M}^{f}$ be dominated. Then there exists $M^{*} \subseteq M$ that is strictly dominated.

Proof: Let $M \subseteq \mathcal{M}^{f}$ be dominated by $M^{\prime} \subseteq \mathcal{M}^{f}$. Then

1. there exists a set of assignments $\widetilde{M} \subseteq \mathcal{M}$ that is frequency equivalent to $M^{\prime}$ and

2. there exists a one-to-one function $f: \widetilde{M} \rightarrow M$ such that

(a) for all $\mu \in \widetilde{M}, \mu$ Pareto dominates or equal to $f(\mu)$, and

(b) there exists $\mu \in \widetilde{M}$ such that $\mu$ Pareto dominates $f(\mu)$.

Let $\widehat{M}=\{\mu \in \widetilde{M}: \mu=f(\mu)\}$. Note that $\widetilde{M} \backslash \widehat{M} \neq \emptyset$. Moreover since the range of $f$ is $M \subseteq \mathcal{M}^{f}$ we have $\widehat{M} \subseteq M \cap \widehat{M}$.

We are now ready to show that $M^{*}=M \backslash \widehat{M}$ is strictly dominated. Let $g: \widetilde{M} \backslash \widehat{M} \rightarrow M^{*}$ be such that

$$
\forall \mu \in \widetilde{M} \backslash \widehat{M} \quad g(\mu)=f(\mu)
$$

Then

$$
\forall \mu \in \widetilde{M} \backslash \widehat{M} \quad \mu \text { Pareto dominates } g(\mu)
$$

Moreover since $\widetilde{M}$ is frequency equivalent to $M^{\prime} \subseteq \mathcal{M}^{f}, \widehat{M} \subset M \subseteq \mathcal{M}^{f}$ implies

$$
\forall h \in H \quad \sum_{\mu \in \widetilde{M} \backslash \widehat{M}}\left|\mu^{-1}(h)\right|=|\widetilde{M} \backslash \widehat{M}|
$$

and therefore by Lemma 1 there exists a set of feasible assignments $M^{\prime \prime} \subseteq \mathcal{M}^{f}$ such that $\widetilde{M} \backslash \widehat{M}$ is frequency equivalent to $M^{\prime \prime}$. This together with relation (11) implies that $M^{\prime \prime}$ strictly dominates $M^{*} \subseteq M$.

In order to prove Lemma 1, we need the following well-known result as well as Lemma 0 :

Hall's Theorem (Hall [11]): ${ }^{11}$ Fix $H(I) \subseteq H$ for each $I \subseteq N$. A feasible assignment $\mu \in \mathcal{M}^{f}$ with

$$
\forall I \subseteq N \quad \bigcup_{i \in I} \mu(i) \subseteq H(I)
$$

exists if and only if $|H(I)| \geq|I|$ for all $I \subseteq N$.

\footnotetext{
${ }^{11}$ See also Roth and Sotomayor [21].
} 
Lemma 0: Let $\widetilde{M}=\left\{\tilde{\mu}_{1}, \tilde{\mu}_{2}, \ldots, \tilde{\mu}_{k}\right\} \subset \mathcal{M}$ be a set of assignments such that

$$
\forall h \in H \quad \sum_{t=1}^{k}\left|\tilde{\mu}_{t}^{-1}(h)\right|=k
$$

Then there exists a feasible assignment $\mu \in \mathcal{M}^{f}$ such that

$$
\forall i \in N \quad \mu(i) \in \bigcup_{t=1}^{k} \tilde{\mu}_{t}(i)
$$

Proof: Let $I=\left\{i^{1}, \ldots, i^{m}\right\}$ be an arbitrary subset of agents and let

$$
H(I)=\bigcup_{\ell=1}^{m} \bigcup_{t=1}^{k} \tilde{\mu}_{t}\left(i^{\ell}\right)
$$

Thanks to Hall's theorem all that needs to be shown is that $|I|=m \leq H(I) .{ }^{12}$

Suppose $m>|H(I)|$. Two simple counting exercises yield the desired contradiction. First we have

$$
\left|\bigsqcup_{\ell=1}^{m} \bigsqcup_{t=1}^{k} \tilde{\mu}_{t}\left(i^{\ell}\right)\right|=\left|\bigsqcup_{h \in H(I)} \bigsqcup_{t=1}^{k}\left(\tilde{\mu}_{t}^{-1}(h) \cap I\right)\right|
$$

Next we have

$$
\left|\bigsqcup_{\ell=1}^{m} \bigsqcup_{t=1}^{k} \tilde{\mu}_{t}\left(i^{\ell}\right)\right|=k \cdot m
$$

whereas

$$
\left|\bigsqcup_{h \in H(I)} \bigsqcup_{t=1}^{k}\left(\tilde{\mu}_{t}^{-1}(h) \cap I\right)\right|=\sum_{h \in H(I)}\left|\sum_{t=1}^{k}\right| \tilde{\mu}_{t}^{-1}(h) \cap I|| \leq \sum_{h \in H(I)} k=k .|H(I)|<k . m
$$

\footnotetext{
${ }^{12}$ Alternatively one can show that the following recursive construction yields one such feasible assignment $\mu \in \mathcal{M}^{f}:$
}

Let $i^{1} \in N$ be such that

$$
\forall i \in N \quad\left|\bigcup_{t=1}^{k} \tilde{\mu}_{t}\left(i^{1}\right)\right| \leq\left|\bigcup_{t=1}^{k} \tilde{\mu}_{t}(i)\right|
$$

Pick $h^{1} \in \bigcup_{t=1}^{k} \tilde{\mu}_{t}\left(i^{1}\right)$ and set $\mu\left(i^{1}\right)=h^{1}$.

In general, given $\left(i^{1}, h^{1}\right),\left(i^{2}, h^{2}\right), \ldots,\left(i^{\ell-1}, h^{\ell-1}\right)$, let $i^{\ell} \in N$ be such that

$$
\forall i \in N \backslash\left\{i^{l}, \ldots, i^{\ell-1}\right\} \quad\left|\left[\bigcup_{t=1}^{k} \tilde{\mu}_{t}\left(i^{\ell}\right)\right] \backslash\left\{h^{1}, \ldots, h^{\ell-1}\right\}\right| \leq\left|\left\lceil\bigcup_{t=1}^{k} \tilde{\mu}_{t}(i)\right] \backslash\left\{h^{1}, \ldots, h^{\ell-1}\right\}\right|
$$

Pick $h^{\ell} \in \bigcup_{t=1}^{k} \tilde{\mu}_{t}\left(i^{\ell}\right) \backslash\left\{h^{1}, \ldots, h^{\ell-1}\right\}$ and set $\mu\left(i^{\ell}\right)=h^{\ell}$. 
contradicting (12), (13) and completing the proof.

Lemma 1: Let $\widetilde{M}=\left\{\tilde{\mu}_{1}, \tilde{\mu}_{2}, \ldots, \tilde{\mu}_{k}\right\} \subset \mathcal{M}$ be a set of assignments such that

$$
\forall h \in H \quad \sum_{t=1}^{k}\left|\tilde{\mu}_{t}^{-1}(h)\right|=k .
$$

Then there exists a set of feasible assignments $M^{\prime}=\left\{\mu_{1}^{\prime}, \mu_{2}^{\prime}, \ldots, \mu_{k}^{\prime}\right\} \subset \mathcal{M}^{f}$ such that $\widetilde{M}$ is frequency equivalent to $M^{\prime}$.

Proof: By induction. For $k=1$ the proof is obvious.

Assume that the claim is correct for $k=r$. Let $\widetilde{M}=\left\{\tilde{\mu}_{1}, \ldots, \tilde{\mu}_{r}, \tilde{\mu}_{r+1}\right\} \subset \mathcal{M}$ be such that

$$
\forall h \in H \quad \sum_{t=1}^{r+1}\left|\tilde{\mu}_{t}^{-1}(h)\right|=r+1
$$

Then by Lemma 0 , we can always find a feasible assignment $\mu \in \mathcal{M}^{f}$ such that

$$
\forall i \in N \quad \mu(i) \in \bigcup_{t=1}^{r+1} \tilde{\mu}_{t}(i)
$$

For each $i \in N$, let $\ell_{i} \in\{1, \ldots, r+1\}$ be such that $\mu(i)=\tilde{\mu}_{\ell_{i}}(i)$. Next construct the set of assignments $\widehat{M}=\left\{\hat{\mu}_{1}, \ldots, \hat{\mu}_{r}\right\}$ as follows:

$$
\forall \ell \in\{1, \ldots, r\} \quad \hat{\mu}_{\ell}(i)= \begin{cases}\tilde{\mu}_{\ell}(i) & \text { if } \ell \neq \ell_{i} \\ \tilde{\mu}_{r+1}(i) & \text { if } \ell=\ell_{i}\end{cases}
$$

By construction

$$
\forall h \in H \quad \sum_{t=1}^{r}\left|\hat{\mu}_{t}^{-1}(h)\right|=r
$$

and therefore by the induction hypothesis, there exists a set of feasible assignments $M=$ $\left\{\mu_{1}, \ldots, \mu_{r}\right\}$ such that $\widehat{M}$ is frequency equivalent to $M$. But then, $\widetilde{M}$ is frequency equivalent to $M^{\prime}=M \cup\{\mu\}$.

\section{References}

[1] A. Abdulkadiroğlu and T. Sönmez, Random serial dictatorship and the core from random endowments in house allocation problems, Econometrica 66 (1998), 689-701.

[2] A. Abdulkadiroğlu and T. Sönmez, House allocation with existing tenants, J. Econ. Theory 88 (1999), 233-260. 
[3] A. Bogomolnaia and H. Moulin, A new solution to the random assignment problem, J. Econ. Theory 100 (2001), 295-328.

[4] A. Bogomolnaia and H. Moulin, A simple random assignment problem with a unique solution, Econ. Theory 19 (2002), 623-635.

[5] H. Crès and H. Moulin, Scheduling with opting out: Improving upon random priority, Oper. Res. 49 (2001), 565-577.

[6] L. Ehlers, "Coalitional strategy-proof house allocation," mimeo, Maastricht University, 2000, forthcoming in J. Econ. Theory.

[7] H. Ergin, Consistency in house allocation problems, J. Math. Econ. 34 (2000), 77-97.

[8] D. Gale and L.S. Shapley, College admissions and stability of marriages, Amer. Math. Monthly 69 (1962), 9-15.

[9] R. Garratt and C-Z. Qin, Cores and competitive equilibria with indivisibilities and lotteries, J. Econ. Theory 68 (1996), 531-543.

[10] J. Hadar and W. Russell, Rules for ordering uncertain prospects, Amer. Econ. Rev. 59 (1969), 25-34.

[11] P. Hall, On representatives of subsets, J. London Math. Soc. 10 (1935), 26-30.

[12] A. Hylland and R. Zeckhauser, The efficient allocation of individuals to positions, $J$. Polit. Econ. 87 (1979), 293-314.

[13] S. Karlin, Dynamic inventory policy with varying stochastic demands, Management Sci. 6 (1960), 231-258.

[14] A. Mas-Colell, M.D. Whinston and J.R. Green, "Microeconomic Theory," Oxford University Press, New York-Oxford, 1995.

[15] A. McLennan, "Ordinal efficiency and the polyhedral separating hyperplane theorem," mimeo, University of Minnesota, 1999, forthcoming in J. Econ. Theory.

[16] E. Miyagawa, Strategy-proofness and the core in house allocation problems, Games Econ. Behav. 38 (2002), 347-361.

[17] S. Papai, Strategyproof assignment by hierarchical exchange, Econometrica 68 (2000), 1403-1433.

[18] J.P. Quirk and R. Saposnik, Admissibility and measurable utility functions, Rev. Econ. Stud. 29 (1962), 140-146. 
[19] A.E. Roth and U.G. Rothblum, Truncation strategies in matching markets: In search of advice for participants, Econometrica 67 (1999), 21-43.

[20] A.E. Roth, U.G. Rothblum and J.H. Vande Vate, Stable matchings, optimal assignments, and linear programming, Math. Oper. Res. 18 (1993), 803-828.

[21] A.E. Roth and M. Sotomayor, "Two-Sided Matching: A Study in Game-Theoretic Modeling and Analysis," Cambridge University Press, Cambridge, 1990.

[22] L. Shapley and H. Scarf, On cores and indivisibility, J. Math. Econ. 1 (1974), 23-37.

[23] L.-G. Svensson, Strategy-proof allocation of indivisible goods, Soc. Choice Welfare 16 (1999), 557-567.

[24] L. Zhou, On a conjecture by Gale about one-sided matching problems, J. Econ. Theory 52 (1990), 123-135. 